\title{
Has Convalescent Plasma Therapy Hesitancy Increased COVID-19 Mortality?
}

\author{
Purvish M. Parikh ${ }^{1} \quad$ T. Sood ${ }^{2} \quad$ V. Agarwala ${ }^{3} \quad$ S. Kate ${ }^{4}$ \\ ${ }^{1}$ Mumbai Oncocare Centers, Mumbai, Maharashtra, India \\ 2Department of Medical Oncology, Manipal Hospital, Bengaluru, \\ Karnataka, India \\ ${ }^{3}$ Department of Medical Oncology, Narayana Superspeciality \\ Hospital, Kolkata, West Bengal, India \\ ${ }^{4}$ Department of Medical Oncology, HCG Manavata Cancer Center \\ Nashik, Maharashtra, India
}

Address for correspondence Purvish M. Parikh, MD, DNB,FICP, PhD, ECMO, CPI, MBA, Department of Medical Oncology, Mumbai Oncocare Centers, 1st Floor, Blue Nile Building, LBS Road, Thane, Maharashtra 400601, India (e-mail: Purvish1@gmail.com).

\section{South Asian J Cancer 2021;10:42-45.}

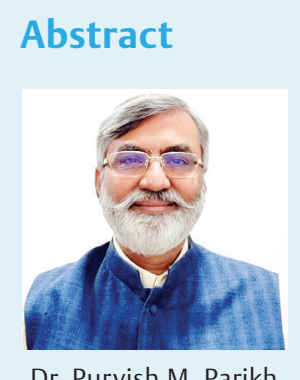

Dr. Purvish M. Parikh
We bring to you our viewpoint and a snapshot of the journey of convalescent plasma therapy (CPT) in the management of ongoing coronavirus disease 2019 (COVID-19) pandemic. We also discuss how best to use the updated data on this important treatment option and maximize benefit for our patients, thereby saving lives, especially in resource constraint settings.

Keywords

pandemic, SARS-CoV-2, antibodies, immunity, titer, donor

\section{Introduction}

The fact that convalescent plasma therapy (CPT) has medicinal value is nothing new. Its use commenced more than 100 years ago. ${ }^{1}$ The simple principle is that the blood of a previously infected individual (who has now recovered) has circulating antibodies which can be given to another individual (after precautions and testing) with the hope that the protective antibodies will be able to target the same infective agent, rescue from acute and fatal consequences, and hasten the recovery of the second patient.

The ongoing coronavirus disease 2019 (COVID-19) pandemic resulted in renewed interest in this form of therapy, but initial results were disappointing. The emergence of virulent mutated variations of COVID-19 and the second wave has forced us to relook at this treatment option. ${ }^{2}$

A simple online search using the terms "convalescent plasma therapy COVID-19" gives interesting results. There were $22,20,000$ hits on google, 18,200 references on google scholar, and 820 articles on PubMed. ${ }^{3-5}$ When we added the words "randomized trial" to the above search terms, we got 74 (for 2020) and 43 (for 2021) results on PubMed. ${ }^{5}$

\section{DOI https://doi.org/10.1055/s-0041-1733351 ISSN 2278-330X}

How to cite this article: Parikh P. M, Sood T, Agarwala V, et al. Has Convalescent Plasma Therapy Hesitancy Increased COVID-19 Mortality? South Asian J Cancer 2021;10(1):42-45.

\section{Negative Studies}

The first study was reported in JANA in 2020 and included data from seven centers in Wuhan, China. ${ }^{6}$ Patients were enrolled between February 14, 2020 and April 1 ${ }^{\text {st }}, 2020$. All patients had severe or life threatening COVID. While the initial plan was to enroll 200 patients, only 103 could be included. They found no difference in day 28 mortality (15.7 vs. $24.0 \%$; $p=0.30$ ) or time of discharge ( 51.0 vs. $36.0 \%$ discharged by day 28; hazard ratio, $1.61[p=0.12])$. The advantage they identified was a better rate of RT PCR negativity at 72 hours, i.e., $87.2 \%$ of the convalescent plasma group vs. $37.5 \%$ of the control group (OR, 11.39 [95\% CI, 3.91-33.18]; $p<0.001$ ).

Another negative study was reported by Ventura et al. ${ }^{7}$ In their trial, 228 patients were assigned to receive convalescent plasma and 105 to receive placebo. The overall mortality was $10.96 \%$ in the convalescent plasma group and $11.43 \%$ in the placebo group ( $95 \% \mathrm{CI},-7.8$ to $6.8 ; p=\mathrm{NS})$.

Several other studies (in United States, Spain, United Kingdom, China, Netherlands) could not complete intended enrolment and had to close prematurely, including the C3PO NIH Trial (enrolled 511/900 patients). ${ }^{8}$ The U.S. FDA sponsored program at Mayo Clinic enrolled more

(C) 2021. MedIntel Services Pvt Ltd.

This is an open access article published by Thieme under the terms of the Creative Commons Attribution-NonDerivative-NonCommercial-License, permitting copying and reproduction so long as the original work is given appropriate credit. Contents may not be used for commercial purposes, or adapted, remixed, transformed or built upon. (https://creativecommons.org/licenses/by-nc-nd/4.0/).

Thieme Medical and Scientific Publishers Private Ltd A-12, Second Floor, Sector -2, NOIDA -201301, India 
than 1,00,000 patients. Though many patients improved clinically, the exact role of CPT was clouded by confounding factors like simultaneous administration of other therapies (antivirals/corticosteroids). They did a retrospective analysis on the subset of 3,082 patients whose neutralizing antibody titer data was available. They concluded that the relative risk of mortality was lower in hospitalized nonventilated patients who received high titer CPT. ${ }^{8}$

An open label, parallel arm, phase II, multicenter, randomized controlled trial conducted in India showed that convalescent plasma was not associated with a reduction in progression to severe COVID-19 or all-cause mortality. ${ }^{9,10}$ However it was noted that CPT was associated with earlier resolution of shortness of breath and fatigue and higher negative conversion of severe acute respiratory syndrome coronavirus 2 (SARS-CoV-2) RNA on day seven of enrolment. This finding opened discussion regarding the use of CPT earlier in disease course, possibly reducing progression to severe disease and acting as a bridge for recovering patients.

No wonder, guidelines could not recommend CPT because of several limitations. Yet, the common thread amongst these reports was that CPT was likely to benefit patients if instituted early when the patients' condition was still not severe. ${ }^{11}$

\section{Turning Point}

On August 23, 2020, U.S. FDA permitted use of CPT for COVID-19 outside the clinical trial through emergency use authorization. ${ }^{12}$

Subsequently it was reported by Casadevall et al in 2021 that the number of CP units dispensed by American blood banks correlated with the number of COVID-19 patients in that country. ${ }^{13}$

\section{Positive Studies}

Eric Salazar and colleagues published their cohort data wherein the Kaplan-Meier curves for mortality within 60 days (day 0 is day of receiving plasma with an antireceptor binding domain IgG titer $\geq 1: 1,350$ ) showed impressive benefit. ${ }^{14}$ Unfortunately, such "ideal world" protective titers were seen in less than $5 \%$ of potential donors.

Jim et al also recently published data on the neutralizing antibody titer in their 250 consecutive patients. ${ }^{15}$ They measured plasma titers at a median of 67 days after onset of symptoms, giving adequate time to the body to mount an appropriate immune response. Interestingly only $60 \%$ had neutralizing antibody titer of more than 1:80 in their publication whereas Ling et al detected almost all (93\%) individuals had titers of more than 1:640. ${ }^{16}$ Higher titers correlate with older age, male gender, and those who had fever and/or hospitalization at the time of having their COVID-19 infection. Their data also showed that the neutralizing antibody titers decline within approximately 90 to 100 days, suggesting the right time to utilize potential donors. This is the reason the cut-off for acceptable CPT donors has been revised internationally. ${ }^{17}$

Very interesting is the manuscript by Anand et al, accepted for publication in Cell Reports Medicine. ${ }^{18}$ It includes the study related to the humoral immunity response against COVID-19 spike protein over a period of more than 31 weeks ( - Fig. 1). This data will act as a backbone for future studies as well as the management strategies in overcoming the COVID-19 pandemic.

A four-center study from Kuwait was conducted between May 21st and June $30^{\text {th }}, 2020 .{ }^{19}$ This was a nonrandomized but prospective cohort study. The CPT arm had 135 cases and control arm (standard treatment for COVID-19 without CPT) included 233 patients. All the 368 patients had moderate to severe COVID-19 disease. Patients with severe COVID-19 had faster clinical improvement ( 7 days in CPT arm vs. 15.5 days in control group [ $p=0.003]$ ). Moderate COVID-19 patients in study arm had significantly lower 30-day mortality rate. All patient treated with CPT had faster $\mathrm{O}_{2}$ saturation improvement; faster lymphocyte count improvement; and faster C-reactive protein decline. There were no serious transfusion reactions.

Another randomized, double-blind, placebo-controlled trial was conducted of convalescent plasma with high IgG titers against SARS-CoV-2 in older adult patients..$^{20}$ Administration of CPT was within 72 hours of onset of mild COVID-19 symptoms. A total of 160 patients underwent randomization. In the intention-to-treat population, severe respiratory disease developed in 13 of 80 patients (16\%) who received convalescent plasma and 25 of 80 patients (31\%) who received placebo with a relative risk reduction of $48 \%$.

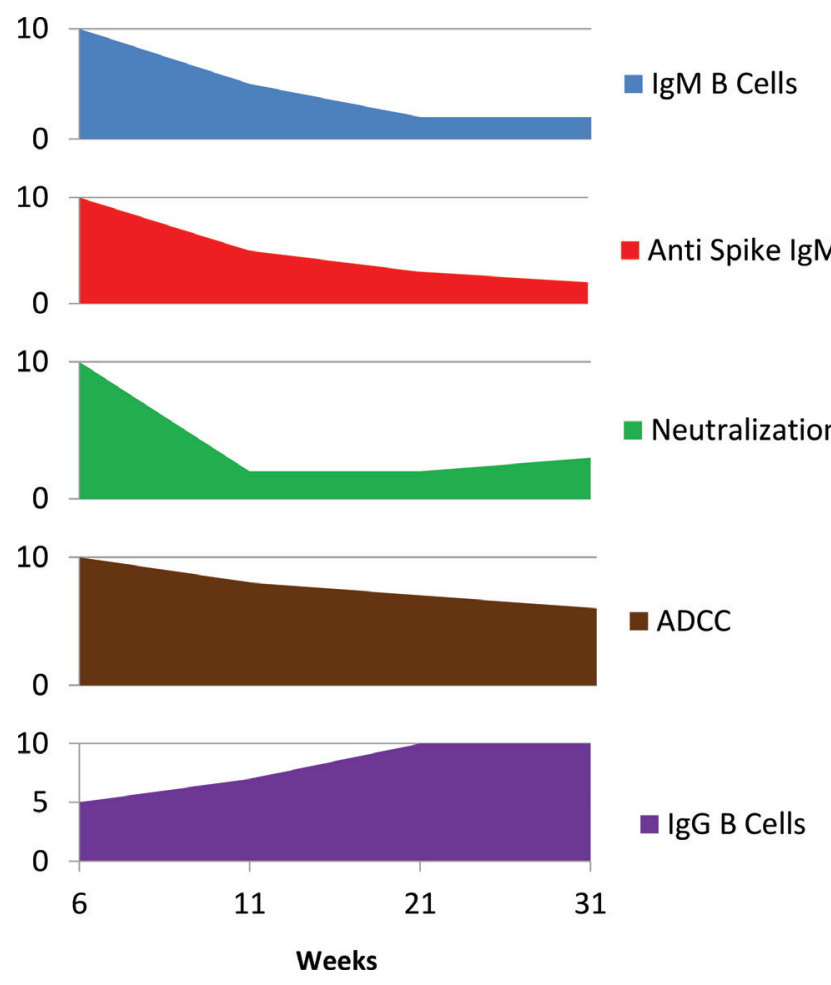

Fig. 1 Anti COVID-19 spike humoral responses. ${ }^{18}$ 
An observational study led by Budhiraja et al, evaluated 333 patients that received plasma therapy during COVID-19 treatment. ${ }^{21}$ Among patients with COVID-19 admitted to ICU, mortality was significantly lower in plasma group ( 25.5 vs. $33.2 \% ; p=0.026$ ). The study concluded that the use of CPT reduced mortality in patients admitted to ICU who were older than 60 years, females, those with comorbidities, and especially those who required some form of ventilation.

\section{Indian Initiatives}

Currently India is in the midst of a massive second wave of the COVID-19 pandemic. ${ }^{22}$ All stake holders have ramped up their efforts to tackle the increasing load of sick patients who require significant treatment. ${ }^{23}$

Amberhealth.in is one such volunteer run nonprofit initiative based out of Bangalore set up by Dr. Tanvi Sood and Nikhil Joy. ${ }^{24}$ Operational since the first wave in India, it was launched in mid-August around the same time as USFDA EUA approval for plasma therapy for COVID-19 management. It was started to provide a digital platform for COVID-19 plasma donation facilitation. It is operated by members with medical background and technical expertise to counsel potential donors, answer frequently asked questions, and build relations with existing plasma banks across hospitals in the country. It focuses on helping voluntary plasma donors get connected with licensed plasma donor centers for a safe and smooth plasma donation experience and also with recipient requests through verified channels in times of emergency.

The peak months of operation lasted till November 2020 after which donors reduced as active cases saw a dip in India. Amberhealth.in has received 63 registrations from willing potential donors till date of which 43 donors cleared the initial eligibility criteria (age 18-60 years, weight $>50 \mathrm{~kg}$, nullipara women, no comorbidities, 28 days post recovery from last symptom or 14 days post COVID RT PCR negative test). These 43 donors were personally facilitated to plasma banks for the elective donation process or connected directly to families looking for plasma urgently for patients. The donation process collects 2 units of $220 \mathrm{~mL}$ plasma per donor. The second level of eligibility is determined at the actual site of donation during which further six donors were deemed ineligible based on low antibody titer $(<1: 80)$ or low hemoglobin levels ( $<12.5 \mathrm{mg} / \mathrm{dL}$ ). Of the 43 donors, 37 were eligible with high titer antibodies and their plasma collection was completed. Data was followed up through the families on the outcomes of the patients which were connected directly to donors facilitated through our initiative. Of the 26 recipients, 10 patients had moderate severity of the disease and the remaining 16 had severe disease. All the patients were receiving steroids and 21 patients were receiving Remdesivir. Seven patients succumbed to their disease (all of them belonged to the severe disease category, on NIV or ventilator). Plasma therapy has been used as an affordable treatment option for patients with steroid refractory hypoxia, possibly helping in reducing the recovery time and progression to invasive ventilation. The resurgence of the second wave from March 2020 has seen an increasing demand for plasma. While availability of eligible donors lags behind its demand, involvement of well-known personalities like Bhoomi Pednekar leads to a quantum jump in awareness amongst the general public and thus a ramp up in donor availability is expected. ${ }^{25}$

\section{Take Home Messages}

1. Convalescent plasma therapy (CPT) is not new-having been used by medical professionals since more than 100 years.

2. There are more than xxx publications in PubMed regarding CPT for coronavirus disease 2019 (COVID-19).

3. Earlier clinical trials were unsuccessful in delineate true benefit of CPT because we had limited knowledge about its application; They were used in patients at the time when COVID-19 had become serious; and results were confounded by other medications being given to the patients.

4. Several prospective clinical trials could not be completed because of insufficient enrolment-especially during the first wave of the pandemic.

5. U.S. FDA approval of CPT use outside a clinical trial setting as emergency use authorization was a watershed turning point in the application of CPT for COVID-19.

6. More recent clinical trials have shown most appropriate use of CPT.

7. Patients should be selected early in their phase of the COVID-19 infection-before they have significant reduction in $\mathrm{SiO}_{2}$ or require ICU care. For LMIC, CPT is a cheaper alternative that can save lives amongst patients who are not eligible for or cannot take more expensive antiviral, antibodies, etc.

8. With increasing population already being vaccinated, both the incidence and severity of COVID-19 is likely to fall. Having said that, if previously vaccinated patients are being considered for CPT, it would be prudent to check their neutralizing antibody titers first. CPT should be given only to those whose titers are not elevated.

9. Appropriate donor selection should be based on neutralizing antibody titers. Older male persons who have recovered from COVID-19 infection that was associated with fever for 3 days and/or required hospitalization can be used as clinical criteria to enrich eligible donor selection.

10. Several initiatives from India have pioneered the use of CPT to the benefit of thousands of COVID-19 patients. Amberhealth.in and Bhoomi Pednekar taking up this cause are only two such examples.

11. With more than 100,000 patients having received CPT in United States alone, we now have published data from more than 20,000 patients. Serious adverse events are seen in less than $1 \%$ of instances and most of them are found to be unrelated to CPT, reinforcing their safety. 
Conflict of Interest

None declared.

\section{References}

1 Available at: https://www.youtube.com/watch?v=SaaeJC1qAUM Accessed May 9th, 2021

2 Parikh PM, Joshi S, Gulia A. Resurgence of COVID-19 in Indiachallenges and solutions. Int J Med Sci 2021;73:70-74

3 Available at: https://www.google.com/search?q=convalescent+plasma+therapy+covid-19. Accessed May 9th, 2021

4 Available at: https://scholar.google.com/scholar?hl=en\&as_ $\mathrm{sdt}=0 \% 2 \mathrm{C} 5 \& \mathrm{q}=$ convalescent+plasma+therapy+covid$19 \& \mathrm{btnG}=$. Accessed May 9th, 2021

5 Available at: https://pubmed.ncbi.nlm.nih.gov/?term=convalsecent+plasma+therapy+covid-19+202. Accessed May 9th, 2021

6 Li L, Zhang W, Hu Y, et al. Effect of convalescent plasma therapy on time to clinical improvement in patients with severe and life-threatening COVID-19: a randomized clinical trial. JAMA 2020;324(5):460-470

7 Simonovich VA, Burgos Pratx LD, Scibona P, et al. PlasmAr Study Group. A randomized trial of convalescent plasma in Covid-19 severe pneumonia.NEnglJMed2021;384(7):619-629

8 COVID-19 resources. Available at: https://www.hematology. org/covid-19/covid-19-and-convalescent-plasma. Version 7.0; last updated March 23, 2021. Accessed May 9th, 2021

9 Agarwal A, Mukherjee A, Kumar G, Chatterjee P, Bhatnagar T, Malhotra P; PLACID Trial Collaborators. Convalescent plasma in the management of moderate covid-19 in adults in India: open label phase II multicentre randomised controlled trial (PLACID Trial. BMJ 2020;371:m3939

10 Gundavda KK, Parikh PM. Convalescent plasma therapy for COVID-19 still has the potential to save lives-the ICMR PLACID study dissected. Int J Molecular \& Immuno Oncology 2020;6:47-49

11 Sarkar S, Soni KD, Khanna P. Convalescent plasma is a clutch at straws in COVID-19 management! A systematic review and meta-analysis. J Med Virol 2021;93(2):1111-1118

12 Pau AK, Aberg J, Baker J, et al. Convalescent plasma for the treatment of COVID-19: perspectives of the National Institutes of Health COVID-19 treatment guidelines panel. Ann Intern Med 2021;174(1):93-95
13 Casadevall A, Dragotakes Q Johnson PW, et al. Convalescent plasma use in the United States was inversely correlated with COVID-19 mortality: did plasma hesitancy cost lives? MedRxiv doi: https://doi.org/10.1101/2021.04.07.21255089/prpt

14 Salazar E, Christensen PA, Graviss EA, et al. Significantly decreased mortality in a large cohort of coronavirus disease 2019 (COVID-19) patients transfused early with convalescent plasma containing high-titer anti-severe acute respiratory syndrome coronavirus 2 (SARS-CoV-2) spike protein IgG. Am J Pathol 2021;191(1):90-107

15 Boonyaratanakornkit J, Morishima C, Selke S, et al. Clinical, laboratory, and temporal predictors of neutralizing antibodies against SARS-CoV-2 among COVID-19 convalescent plasma donor candidates. J Clin Invest 2021;131(3):e144930

16 Li L, Tong X, Chen H, et al. Characteristics and serological patterns of COVID-19 convalescent plasma donors: optimal donors and timing of donation. Transfusion 2020;60(8):1765-1772

17 Jungbauer C, Weseslindtner L, Weidner L, et al. Characterization of 100 sequential SARS-CoV-2 convalescent plasma donations. Transfusion 2021;61(1):12-16

18 Anand SP, Prévost J, Nayrac M, et al. Longitudinal analysis of humoral immunity against SARS-CoV-2 Spike in convalescent individuals up to 8 months post-symptom onset. Cell Rep Med 2021;2(6):100290

19 Alsharidah S, Ayed M, Ameen RM, et al. COVID-19 convalescent plasma treatment of moderate and severe cases of SARS-CoV-2 infection: a multicenter interventional study. Int J Infect Dis 2021;103:439-446

20 Libster R, Pérez Marc G, Wappner D, et al. Fundación INFANT-COVID-19 Group. Early high-titer plasma therapy to prevent severe Covid-19 in older adults. N Engl J Med 2021;384(7):610-618

21 Budhiraja S, Dewan A, Aggarwal R, et al. Effectiveness of convalescent plasma in Indian patients with COVID-19. Blood Cells Mol Dis 2021;88:102548

22 Parikh P, Singh R. Hot race for COVID-19 vaccines-light at the end of the tunnel just got brighter. Int J Mol Immuno Oncol 2021;6(1):1-3

23 Raja T, Parikh PM, Ghafur A, et al. South Asian Declarationconsensus guidelines for COVID-19 vaccination in cancer patients. South Asian J Cancer 2021; In press

24 Available at: www.amberhealth.in. Accessed May 9, 2021

25 Available at: https://www.youtube.com/watch?v=VnKiviXr59M. Accessed May 9, 2021 